\title{
The genetic basis of cis-regulatory divergence between the subspecies of cultivated rice (Oryza sativa)
}

Malachy T Campbell ${ }^{1}$, Qian Du ${ }^{2}$, Kan Liu ${ }^{2}$, Sandeep Sharma ${ }^{3}$, Chi Zhang ${ }^{2}$, Harkamal Walia ${ }^{1 *}$

${ }^{1}$ Department of Agronomy and Horticulture, University of Nebraska Lincoln, Lincoln, NE, USA 68583

${ }^{2}$ School of Biological Sciences, University of Nebraska Lincoln, Lincoln, NE, USA 68583

${ }^{3}$ Marine Biotechnology and Ecology Division, CSIR-CSMCRI, Bhavnagar, Gujarat, India

\section{*Corresponding Author:}

Harkamal Walia

Department of Agronomy and Horticulture

University of Nebraska Lincoln

Lincoln, Nebraska 68583

hwalia2@unl.edu

\section{Running title: Genetics of cis-regulatory divergence in rice}

Keywords: Transcriptome, rice, expression QTL, genome-wide association, genomics 


\section{Abstract}

Cultivated rice consists of two subspecies, Indica and Japonica, that exhibit well-characterized differences at the morphological and genetic levels. However, the differences between these subspecies at the transcriptome level remains largely unexamined. Here, we provide a comprehensive characterization of transcriptome divergence and cis-regulatory variation within rice using transcriptome data from 91 accessions from a rice diversity panel (RDP1). The transcriptomes of the two subspecies of rice are highly divergent. The expression and genetic diversity was significantly lower within Japonica relative to Indica, which is consistent with the known population bottleneck during Japonica domestication. Moreover, 1,860 and 1,325 genes showed differences in heritability in the broad and narrow sense respectively, between the subspecies, which was driven largely by environmental and genetic effects rather than differences in phenotypic variability. We leveraged high-density genotypic data and transcript levels to identify cis-regulatory variants that may explain the genetic divergence between the subspecies. We identified significantly more eQTL that were specific to the Indica subspecies compared to Japonica, suggesting that the observed differences in expression and genetic variability also extends to cis-regulatory variation. We next explored the potential causes of this cis-regulatory divergence by assessing local genetic diversity for cis-eQTL. Local genetic diversity around subspecies-specific cis-eQTL was significantly lower than genome-wide averages in subspecies lacking the eQTL, suggesting that selective pressures may have shaped regulatory variation in each subspecies. This study provides the first comprehensive characterization of transcriptional and cis-regulatory variation in cultivated rice, and could be an important resource for future studies. 


\section{Introduction}

Cultivated rice consists of two subspecies: Indica and Japonica. Indica varieties are cultivated throughout 2 the tropics, and account for the majority of rice production worldwide. Japonica varieties, on the other hand, 3 are grown in both tropical and temperate environments, and only account for approximately $20 \%$ of rice 4 production.

Although the domestication history of rice remains a contested topic, the most current research collectively suggests that rice was domesticated at least twice from two geographically and ecologically distinct subpopulations of Oryza rufipogon. The unique environmental pressures in these distinct regions, as well as preferences by early farmers for grain characteristics has resulted in large morphological and physiological differences between the two subspecies. These differences have been recognized for centuries, as 10 evidenced by references of Keng and Hsein types of rice found in records from the Han Dynasty in China ${ }_{11}$ (Oka et al., 1991).

The unique natural and agronomic selection pressures placed on the wild progenitors and early proto-domesticates resulted in drastic changes at the genetic level. Work by Huang et al. (2012b) showed 14 considerable reduction in genetic diversity in Indica and Japonica compared with O. rufipogon. Such drastic ${ }_{15}$ reductions in genetic diversity are common following domestication. Moreover, the transition from an 16 out-crossing/heterogamous nature of $O$. rufipogon to the autogamous breeding system of cultivated rice likely led to greater partitioning of genetic diversity among the two subspecies, and further differentiation of the two groups. These large genetic differences have been recognized for nearly a century as hybrids between Indica and Japonica exhibit low fertility (Kato, 1928). More recently, these genetic differences have been realized with the availability of high density molecular markers and full genome sequences for both Indica ${ }_{21}$ and Japonica (Ding et al., 2007; Goff et al., 2002, Yu et al., 2002, Feltus et al., 2004, Stein et al., 2018; Koide 22 et al., 2018, Schatz et al., 2014; Huang et al., 2008; Wang et al., 2014; Huang et al., 2012b). For instance ${ }_{23}$ Ding et al. (2007) showed that approximately $10 \%$ of the genes in the Indica and Japonica genomes showed 24 evidence of presence-absence variation or asymmetrical genomic locations. Several other studies have 25 highlighted genetic differences between the subspecies as structural variants differences, gene acquisition and 26 loss, transposable element insertion and single nucleotide polymorphisms (Goff et al., 2002, Yu et al., 2002, 27 Feltus et al. 2004; Stein et al., 2018, Koide et al., 2018, Schatz et al., 2014; Huang et al., 2008; Wang et al., 28 2014 Huang et al. 2012b).

While the morphological and genetic differences of Indica and Japonica have received considerable 
(Walia et al., 2007, Lu et al., 2010, Jung et al., 2013). Walia et al. (2007) utilized genome-wide expression 32 profiling to characterize the transcriptional responses for two Indica and Japonica cultivars to salinity. This 33 study was performed to elucidate the mechanisms underlying the contrasting responses to stress exhibited by 34 the cultivars, rather than examine the transcriptional difference between the subspecies. Moreover, 35 separating genotypic differences from subspecies differences is not feasible with the low number of cultivars 36 used in these studies. Lu et al. (2010) compared transcriptional profiles of two Indica accessions and a single 37 Japonica accessions and identified many novel transcribed regions, highlighted alternative splicing differences, 38 and differentially expressed genes between accessions. Although these studies provided insights into the 39 transcriptional differences between Indica and Japonica, given the small sample size of the study it has ${ }_{40}$ limited scope for extending conclusions to a population level. Jung et al. (2013) leveraged the large number of public microarray databases to compare transcriptional diversity between the two subspecies. The 983 publicly available Affymetrix microarrays were classified into Indica and Japonica subspecies based on the cultivar name. This study showed that considerable differences in expression levels were evident between the two subspecies. However, considerable information is likely lost due to the heterogeneity in sample types (e.g. $\quad{ }_{45}$ tissue, developmental stage) and varying growth conditions. Thus, a more highly controlled study that ${ }^{46}$ utilized a larger panel with genotypic information would provide greater insight into the differences in ${ }_{47}$ expression levels, as well as provide a mechanism for connecting transcriptional differences between the two ${ }_{48}$ subspecies with genetic variation.

The objective of this study is to examine genetic basis of the transcriptional variation at a population level within the $O$. sativa species. By combining population and quantitative genetics approaches, we aim to elucidate the genetic basis of transcriptional divergence between the two subspecies. To this end, we generated transcriptome data using RNA sequencing on shoot tissue for a panel of 91 diverse rice accession selected from the Rice Diversity Panel1 (RDP1) (Zhao et al., 2011; Famoso et al., 2011; Eizenga et al., 2014). 54 Here, we show that transcriptional diversity between Indica and Japonica subspecies is consistent with diversity at the genetic level. Moreover, we connect transcriptional differences between the two subspecies with divergent patterns of cis-regulatory variation and show that the absence of many cis-regulatory variants are due to unique selective pressures experienced by each subspecies. This study is the first to document the transcriptional divergence between the major subspecies of cultivated rice at a population level, and provides insight into the genetic mechanisms that have shaped this transcriptional divergence. . . . . . 


\section{Materials and Methods}

\section{Plant materials and growth conditions}

[This study used 91 diverse accessions from the Rice Diversity Panel1 (RDP1) (Famoso et al., 2011, Zhao 63 et al. 2011; Eizenga et al., 2014). Seeds were obtained from the USDA-ARS Dale Bumpers Rice Research ${ }_{64}$ Center. The 91 accessions consisted of 13 admixed, 2 aromatic, 9 aus, 23 indica, 21 temperate japonica, and $\quad{ }_{65}$ 23 tropical japonica accessions.

Seeds were dehusked manually and germinated in the dark for two days at $28^{\circ} \mathrm{C}$ in a growth cabinet acclimate them to the conditions in the growth chamber. The seeds were transplanted to 3.25 " x 3.25 " x 5 " pots filled with Turface MVP (Profile Products) in a walk-in controlled environment growth chamber (Conviron). The pots were placed in $36 " \times 24 " \times$ × 8 tubs, that were filled with tap water. Fours days after transplanting the tap water was replaced with half-strength Yoshida solution (Yoshida et al., 1976) (pH 5.8). The $\mathrm{pH}$ of the solution was monitored twice daily and was recirculated from a reservoir beneath the tubs to the growth tubs. The temperatures were maintained at $28^{\circ} \mathrm{C}$ and $25^{\circ} \mathrm{C}$ in day and night respectively and $60 \%$ relative humidity. Lighting was maintained at $800 \mu \mathrm{mol} \mathrm{m}^{-2} \mathrm{~s}^{-1}$ using high-pressure sodium lights (Phillips).

\section{RNA extraction and sequencing}

Ten days after transplant, aerial parts of the seedlings were excised from the roots and frozen immediately in liquid nitrogen. The samples were ground with Tissuelyser II (Invitrogen) and total RNA was isolated with RNAeasy isolation kit (Qiagen) according to manufacturer's instructions. On-column DNAse treatment was performed to remove genomic DNA contamination (Qiagen). Sequencing was performed using Illumina HiSeq 2500. Sixteen RNA samples were combined in each lane. Two biological replicates were used for each accession.

\section{Sequence alignment, expression quantification, and differential expression

Quality control for raw reads was performed using the package FastQC (Andrews et al., 2010). The Illumina 101-bp single-end reads were screened and trimmed using Trimmomatic to ensure each read has average 
multiple locations were discarded (Trapnell et al. 2009, Bolger et al., 2014). The number of reads for each ${ }^{89}$ gene sequence was counted using the HTSeq-count tool with the "union" resolution mode (Anders et al., 90 2015). For down-stream genetic analyses, a variance stabilized transformation was performed on raw read 91 counts to provide approximately homoskedastic values in DEseq2 (Love et al., 2014).

To identify genes that exhibited differential expression between the two subspecies, a mixed linear model was fit that included subspecies as the main fixed effect and accession as a random effect in lme4 (Bates et al. 2015). This 'full' model was compared to a redcued model the lacked subspecies as a fixed effect using a likelihood-ratio test. Prior to differential expression analysis, expression levels were quantile normalized to ensure a Gaussian distribution. Benjamini and Hochberg's method was used to control the false discovery rate, and genes with an FDR $\leq 0.001$ were considered differentially expressed (Benjamini and Hochberg, 1995).

Genes showing differences in presence-absence expression variation (PAV) was determined using a mixed-effects logistic regression model. Briefly, for each sample the expressed genes (number of reads i. 10) 101 were assigned 1 , while those with 10 or less reads were assigned a 0 . A logistic regression model was fit using 102 the 'glmer' function in 'lme4' and included subspecies as a fixed effect and accession as random (Bates et al., ${ }_{103}$ 2015). The significance of the fixed effect of subspecies was determined by comparing the full model above ${ }^{104}$ with a reduced model that lacked subspecies using a likelihood-ratio test. Benjamini and Hochberg's method ${ }_{105}$ was used to control the false discovery rate, and genes with an FDR $\leq 0.001$ were considered as having 106 presence-absence expression variation (Benjamini and Hochberg, 1995).

\section{Subspecies classification}

The 91 accessions were classified into two subspecies using the software STRUCTURE (Pritchard et al., 109 2000 ). Briefly, the software was run using the $44 \mathrm{k}$ SNP data, assuming two subpopulations $(\mathrm{K}=2)$, with ${ }_{110}$ 20000 MCMC replicates and a burn-in of 10000 MCMC replicates.

\section{Expression and genetic diversity analyses}

Principle component analysis of gene expression was conducted for the 91 accessions using 22,675 genes after ${ }_{113}$ variance stabilizing transformation. For, PCA of SNP data the 44k dataset described by Zhao et al. (2011) ${ }_{114}$ was used. SNPs with a MAF $<0.10$ were removed prior to PCA analysis.

The coefficient of variation (CV) was used to estimate the diversity in gene expression within the Indica 116 and Japonica subspecies. Prior to estimating CV genes with low expression (i.e. those with read counts of $\leq{ }_{117}$ 
10 in $\geq 20 \%$ of the samples) were removed, leaving a total of 22,503 genes in Japonica and 21,719 genes in 118 Indica. For the estimation of $\pi$, SNPs were extracted for each subspecies and SNPs with MAF ; 0.05 were 119 excluded. In total 201,891 SNPs were retained for Indica and 161,715 for Japonica. $\pi$ was estimated at each ${ }_{120}$ SNP using the site-pi function in VCFtools (Danecek et al. 2011).

\section{Heritability estimates}

Heritability, both in the broad $\left(H^{2}\right)$ and narrow sense $\left(h^{2}\right)$, was estimated across subspecies for 22,675 genes that were expressed in both Indica and Japonica. To estimate $H^{2}$ a mixed model was fit using lme4 where accession was considered a random effect, and significance of $H^{2}$ was assessed using a restricted likelihood-ratio test in the RLRTsim package (Bates et al., 2015, Scheipl et al., 2008). Benjamini and Hochberg's method was used to control the false discovery rate, and genes with an FDR $\leq 0.001$ were considered to have significant genetic variability (Benjamini and Hochberg, 1995). To assess hertiability in the narrow sense $\left(h^{2}\right)$ a mixed model was fit in asreml-R (Butler et al. 2009). Briefly, a genomic relationship matrix (G) was estimated according to VanRaden (2008) using the approximately 36,901 SNPs described by Zhao et al. (2011). G is estimated as $G=\frac{Z c s Z c s^{\prime}}{m}$, where $Z_{s c}$ is the centered and scaled marker matrix and $m$ is the number of markers. A likelihood-ratio test was used to assess significance and Benjamini and to have significant genetic variability (Benjamini and Hochberg, 1995).

Heritability was assessed within subspecies using the same approaches as described above. However, due to the unequal sample size for the Indica and Japonica subspecies, a random set of 35 Japonica accessions were selected. Genes showing low expression (; 10 reads in ; $20 \%$ of samples) in either subspecies were removed prior analysis, leaving 22,444 genes in Japonica and 22,068 genes in Indica.

\section{Assessing differences in genetic variability between subspecies}

To identify genes showing significant differences in genetic variability $\left(H^{2}\right.$ or $\left.h^{2}\right)$ between subspecies, a permutation approach was used. Here, the 91 accessions were randomly partitioned into two groups of equal 141 size (35 accessions each). Hertiability was estimated as described above and the difference in heritability ${ }_{142}$ between each group was calculated. The resampling approach was repeated 100 times for both $H^{2}$ and $h^{2}$. ${ }^{143}$ This process effectively estimated a null distribution of $\Delta H^{2}$ and $\Delta h^{2}$ values. The heritability estimates for ${ }_{144}$ each subspecies was used to calculate the differences in $H^{2}$ and $h^{2}$ between the two subspecies as ${ }_{145}$ $\Delta H^{2}=H_{J}^{2}-H_{I}^{2}$ or $\Delta h^{2}=h_{J}^{2}-h_{I}^{2}$. These values were compared with the null distribution to assess 
significance.

\section{Joint cis-eQTL analysis}

eQTLs were jointly detected using the eQTL-BMA (Bayesian model averaging) described by Flutre et al. (2013) for 26,675 genes and 274,499 SNPs (MAF > 0.10) McCouch et al. (2016). Prior to eQTL mapping

BLUPs for each gene was calculated and the gene expression level of each gene was transformed into the approach was used within the eQTL-BMA software. Using the eqtlbma_bf program, 10,000 permutations

\section{Detecting evidence of selection at cis-eQTL}

To determine whether the absence of an eQTL was due to of selection, first SNPs from the HDRA dataset within 100kb of each significant eQTL were extracted for the 91 accessions McCouch et al. (2016). For each SNP, nucleotide diversity was determined using the site-pi function in VCFtools and was averaged across the 100kb window (Danecek et al., 2011). Secondly, a genome-wide diversity level was determined for each 
the mean $\pi$ was different from the genome-wide average for each subspecies and class of eQTL.

A similar approach was taken for the $3 \mathrm{~kg}$ data (Alexandrov et al., 2014). For each eQTL SNP, all SNPs $\quad 177$ within $100 \mathrm{~kb}$ of the eQTL SNP was extracted from the 4.8M core SNP data. The MAF was determined for ${ }_{178}$ each of the 12 subpopulations in the $3 \mathrm{~kg}$ data, and SNPs that had low diversity (MAF < 0.01) in 10 of the $12 \quad{ }_{179}$ subpopulations were excluded from further analyses. As above, $\pi$ was calculated for each site. An average $\pi \quad 180$ was determined for each subpopulation at each eQTL by taking the average $\pi$ across the 100kb window. To ${ }_{181}$ obtain a genome wide average, eQTL regions were excluded and $\pi$ was obtained for each subpopulation by $\quad 182$ averaging $\pi$ across the 100kb region. Finally, as above a two-sided Student's $t$-test was performed to assess 183 whether the mean $\pi$ was different from the genome-wide average for each subpopualtion and class of eQTL. ${ }^{184}$ 


\section{Results}

185

We selected 91 accessions to represent

the genetic diversity within Rice Diversity

Panel 1 (RPD1). Using the subpopulation assignment described by Zhao et al. (2011) and Famoso et al. (2011), shoot transcriptome data was generated for 23 tropical japonica, 23 indica, 21 temperate japonica, 13 admixed, 9 aus, and 2 aromatic accessions. Genes with low variance or expression within the expression set were filtered out, as these genes are uninformative for downstream analyses focused on natural variation in gene expression. A total of 25,732 genes were found to be expressed ( $>10$ read counts) in at least one or more of the 91 accessions. This equates to about $46 \%$ of the genes present in the rice genome (total of

A

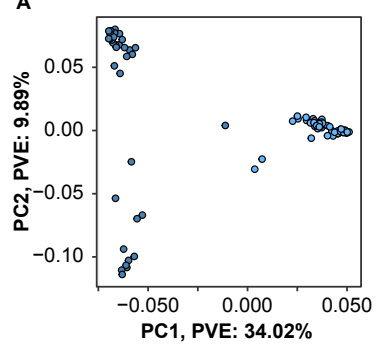

C

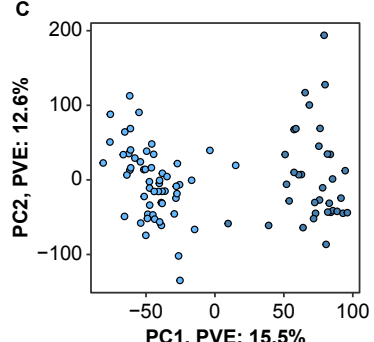

C

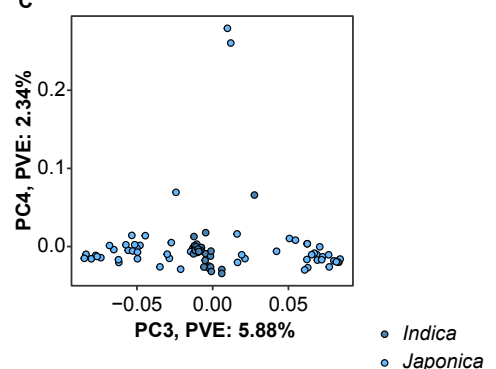

D

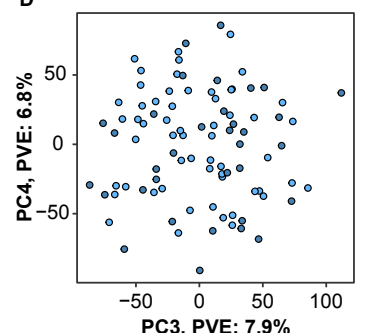

186

187

188

189

190 191 192

Figure 1. Principle component analysis of markers and gene expression matrices. The top four principle components from PCA analysis of the expression data are pictured in A and B to illustrate the divergence of the major subpopulations in rice. The panels in $\mathrm{C}$ and $\mathrm{D}$ summarize PCA of genotypic data. PVE: percent variation explained by each component. 55,986 genes in MSUv7 build).

\section{Divergence between the Indica and Japonica subspecies are evident at the} genetic and transcriptional levels

To examine patterns of variation within the transcriptomics data, we performed principle component analysis were not expressed (< 10 reads) in at least $20 \%$ of the samples. This filtering removed approximately 33,311207 genes, resulting in a total of 22,675 genes that were used for the principal component analysis based on the ${ }_{208}$ normalized read counts. For the genetic analysis, we used 32,849 SNPs. PCA analysis of the expression 209 matrix resulted in a clear separation between the two subspecies along PC1, suggesting a significant transcriptional divergence between Indica and Japonica (Figure 1C,D). The first PC accounted for approximately $26.8 \%$ of the variation in gene expression. While PC1 was able to differentiate between the ${ }^{212}$ two subspecies at the transcriptional level, no clear clustering of accessions was observed along other PCs ${ }_{213}^{213}$ (Figure 11). These results suggest that the the two subspecies of cultivated rice have divergent transcriptomes, ${ }^{214}$ 
but the transcriptomes of the subpopulations are more similar. Consistent with these results observed for ${ }^{215}$

PCs 1 and 2, differentiation between the subspecies was clearly evident along PC1 using the genetic (SNP) 216 data alone (Figure 1A,B). However, the clustering of accessions along PCs 2-4 for the SNP data were ${ }^{217}$ consistent with those described by Zhao et al. (2011) (Figure 1), and were effective in discerning the two ${ }^{218}$ subpopulations in rice. These results collectively suggest that the two subspecies are vastly divergent at ${ }^{219}$ genetic and transcriptional level.

\section{Differential expression analysis reveals contrasting expression between subspecies}

To further explore the differences and identify genes that display divergent expression between the two subspecies, the 91 accessions were first classified into Indica and Japonica-like groups, using the program STRUCTURE with the assumption of two groups and no admixture (Pritchard et al., 2000). A total of 35 accessions were assigned to the Indica subspecies, while 56 were assigned to the Japonica subspecies. Next, a ${ }^{226}$ linear mixed model was fit for each of the 26,675 genes, where subspecies was considered a fixed effect and ${ }^{227}$ accession as a random effect. A total of 7,417 genes were found to exhibit contrasting expression between the ${ }^{228}$ two subspecies (FDR $\leq 0.001$, Supplemental File S1). Of these genes, 4,210 (57\%) showed significantly ${ }_{229}$ higher expression in Japonica relative to Indica, while 3,207 (43\%) showed higher expression in Indica relative to Japonica.

This divergent expression levels observed between the two subspecies could be the result of the presence or absence of genes within the subspecies. To this end, we sought to identify genes showing a presence-absence expression variation (PAV). Genes with a read count greater than 10 were considered as expressed and coded as 1 while those with read counts less than 10 were coded as 0 . These genes were further filtered, so that genes that were expressed in at least $20 \%$, but no more than $80 \%$ of the samples were retained for downstream analyses. A logistic mixed effects model was fit for the 4,263 genes meeting this criteria. In total, 1,980 genes showed evidence of PAV between the two subspecies $(F D R<0.001$; Supplemental File S1). This analysis, enriched for genes that were expressed at higher frequency in Japonica rice compared to Indica. For instance, 1,435 genes were found to be expressed at a significantly greater 236 frequency in Japonica relative to Indica, while only 545 were found to be expressed predominately in Indica. Moreover, we detected significant enrichment for GO terms associated stress response GO:00006950)and response to biotic stress (GO:0009607), as well genes with kinase activity (GO:0016301). Within 
S1). Moreover, 173 were identified

with no evidence of expression in Indica while only 18 were identified in Japonica. Collectively, these results suggest that the divergence between Indica and Japonica subspecies may be due, in part, to differences in mean expression levels as well as presence-absence expression variation.

\section{Japonica}

\section{subspecies exhibits reduced} genetic and transcriptional diversity

Several studies have shown that the unique domestication history of the two subspecies has
A

Figure 2. Genetic and expression diversity within Indica and Japonica accessions. (A) The coefficient of variation was used as an estimate of the diversity in gene expression within each subspecies. A subset of 35 Japonica accessions were randomly selected for diversity analyses to ensure that sample sizes were equal between the two subspecies. The vertical dashed lines represent the mean CV within each subspecies. (B) Site-wise nucleotide diversity $(\pi)$ was used as an estimate of the genetic diversity within each of the subspecies using 36,901 SNPs described by Zhao et al. (2011).

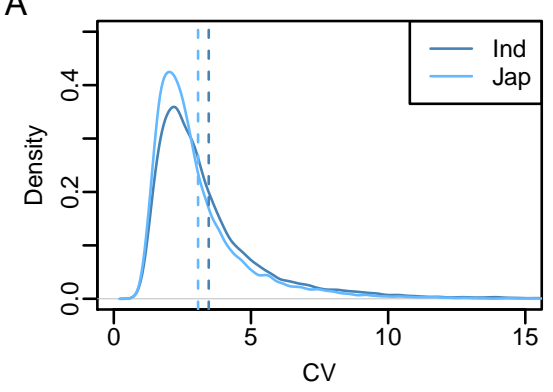

245

246 247 248 249 250 251 more genetically diverse than Japonica Caicedo et al. (2007); Huang et al. (2010, 2012b); Mather et al. ${ }_{258}$ (2007). We next explored the variation in gene expression within each subspecies. Two metrics were used to examine the differences in diversity at both the genetic and transcriptional levels within each subspecies: nucleotide diversity $(\pi)$ and the coefficient of variation (CV). Diversity analyses within each subspecies may be influenced by differences in sample size. Since the number of Japonica accessions were greater than Indica, a subset of 35 Japonica accessions were randomly selected for diversity analyses. The results for the full set of 56 Japonica accessions are provided as Figure S1

Expression diversity was estimated using the coefficient of variation (CV) for 22,675 genes. CV was significantly different between the two subspecies (Wilcoxon rank sum test, $p<0.0001$; Figure 2). The Indica subspecies exhibited approximately $12.6 \%$ higher expression diversity compared to Japonica. On average, CV in the Indica subspecies was 3.46, while in the Japonica subspecies the mean CV was 3.07. These results suggest that the transcriptional diversity is lower in the Japonica subspecies compared to Indica. CV estimates using the complete set of Japonica accession were similar (CV: 3.46 and 3.10 for Indica and Japonica, respectively; Figure S1.

Genetic diversity within each subspecies was estimated using $\pi$ for 33,543 SNPs in randomly selected $35 \quad 272$ Indica and 35 Japonica accessions. Similar differences were observed for $\pi$ as CV, however the differences ${ }^{273}$ between subspecies was much greater (Wilcoxon rank sum test, $p<0.0001$; Figure 2). The Indica subspecies 

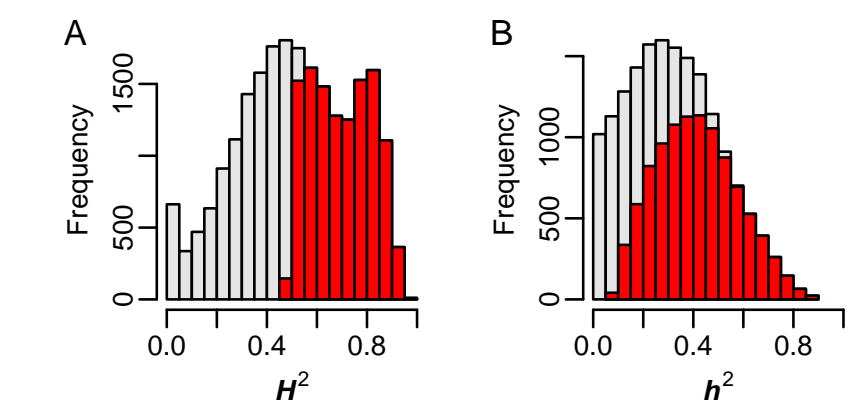

Figure 3. Heritability of gene expression across O. sativa subspecies. Distribution of broad-sense heritability $\left(H^{2}\right)$ and narrow-sense heritability $\left(h^{2}\right)$ for 22,675 genes are pictured in panels A and B, respectfully. Bars highlighted in red indicate genes with significant genetic effects $(F D R<0.001)$.

\section{Gene expression is heritable in cultivated rice}

showed a $64.7 \%$ higher nucleotide diversity $(\pi) \quad 275$ compared to Japonica. On average, $\pi$ estimates $\quad 276$ were 0.26 for Indica and 0.17 for Japonica. These $\quad{ }_{277}$ results are consistent with reports by Huang 278 et al. (2012b) and Garris et al. (2005), and 279 are in agreement with the expression diversity $\quad 280$ reported above. Together these data suggest 281 that the Japonica subspecies exhibits less genetic ${ }_{282}$ and transcriptional diversity compared to Indica. ${ }^{283}$

The above analyses shows a strong differentiation between the subspecies at transcriptional and genetic levels, ${ }^{285}$ and presents a possible linkage between expression and genetic diversity. However, the extent of variation in ${ }^{286}$ gene expression that can be accounted by genetic variation is not yet determined. To estimate the extent to ${ }^{287}$ which variation in gene expression is under genetic control, a mixed model was fit to the expression of each of ${ }^{288}$ the 22,675 genes and the variance between accessions was estimated. The significance of the random between - accession term was determined using a likelihood-ratio test. The broad-sense heritability $\left(H^{2}\right) \quad{ }^{290}$ was estimated as the proportion of the total variance explained by between-accession variance to total ${ }_{291}$ variance. A total of 11,895 genes showed a significant between - accession variance $\left(F D R<0.001 ; H^{2} \geq{ }^{292}\right.$ 0.47), which accounts for approximately $53 \%$ of the genes expressed in at least $20 \%$ of the samples (Figure $2 \mathrm{~A} ; \quad{ }^{293}$ Supplemental File S2). $H^{2}$ ranged from 0.97 to 0.47 , with 4,606 genes showing highly heritable expression ${ }^{294}$ $\left(H^{2}>0.75\right), 7,145$ showing moderate $H^{2}\left(0.5<H^{2} \leq 0.75\right)$, and the remaining 146 showing low $H^{2}$. ${ }_{295}$

To determine the extent to which additive genetic effects could explain variance in gene expression, a 296 genomic relationship matrix was constructed using 32,849 SNPs following VanRaden (2008) and variance ${ }^{297}$ components were estimated using a mixed linear model for each gene. A total of 10,125 genes were identified ${ }^{298}$ with significant $h^{2}$ (Supplemental File S2). Of these, 234 genes had highly heritable expression $\left(h^{2} \geq 0.75\right), \quad 299$ while 2,750 genes showed moderate heritability $\left(0.5 \leq h^{2}<0.75\right)$ (Figure $3 \mathrm{~B}$ ). An additional 7,141 genes 300 showed low narrow sense heritability $\left(h^{2}<0.5\right)$. Collectively, these results indicate that a large portion of 301 the rice transcriptome is under genetic control. 


\section{Genetic variability of gene expression is considerably different between}

The analyses above indicate that the two subpopulations differ at the transcriptional and genetic levels, and that for many genes, variation in expression can be explained by genetic effects. We next asked whether the heritability of gene

expression is different between the two subspecies. To this end, the expression dataset was partitioned into Indica and Japonica subsets and genes with low expression in each subspecies were removed (expressed in less than $20 \%$ of the samples). Since the number of accessions for the two subspecies are unequal, 35 Japonica accessions were randomly sampled to ensure the two samples were of equal size, and the number of genes that were expressed in each subspecies were quantified. Here, a gene was considered expressed if 10 or more reads mapped to the gene in $20 \%$ or more of the samples. A total of 22,444 genes were found to be expressed in at least $20 \%$ of the samples for the Japonica subspecies, while 22,068 were

A

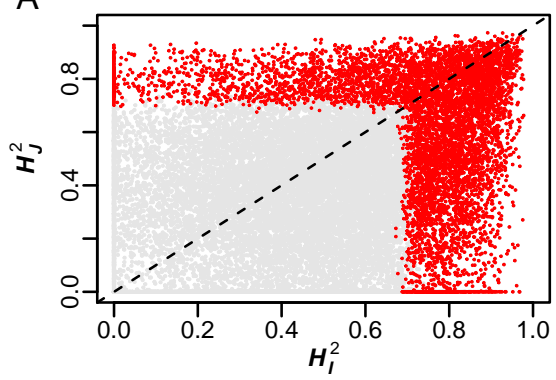

C

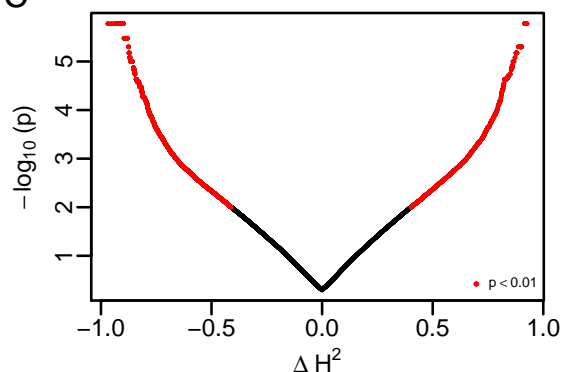

E DPL1 $F \quad$ DPL
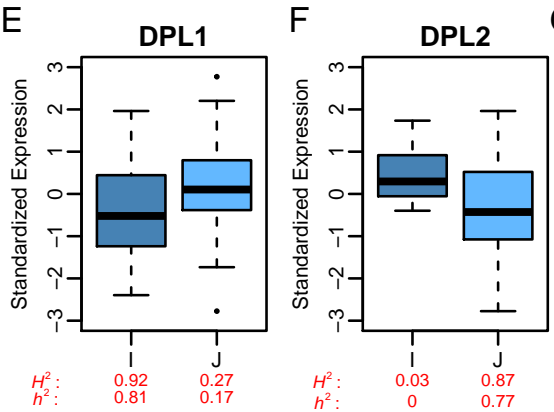

B

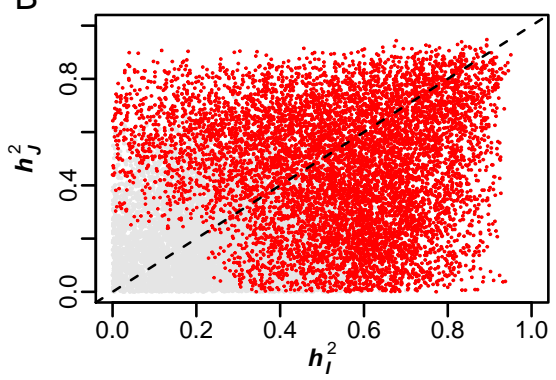

D

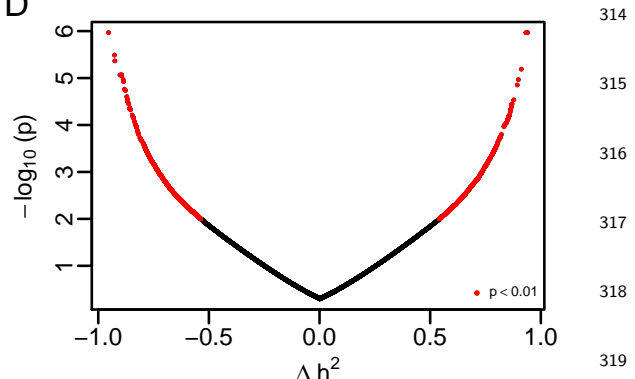

Figure 4. Divergent genetic variability between subspecies. (A) Comparison of broad-sense heritability between Indica $\left(H_{I}^{2}\right)$ and Japonica $\left(H_{J}^{2}\right)$. (B) Comparisons of narrow sense heritability between the two subspecies. Red colored points in B and $\mathrm{C}$ indicate genes with significantly heritable expression $(F D R<0.001)$. Differences in broad $(\mathrm{C})$ and narrow sense heritability (D) between Indica and Japonica. The difference in heritability is calculated as $H_{J}^{2}-H_{I}^{2}$ or $h_{J}^{2}-h_{I}^{2}$. (E-H) Standardized expression of agronomically important genes showing differences in genetic variability between subspecies. The heritability is provided below each box plot. I: Indica, J: Japonica

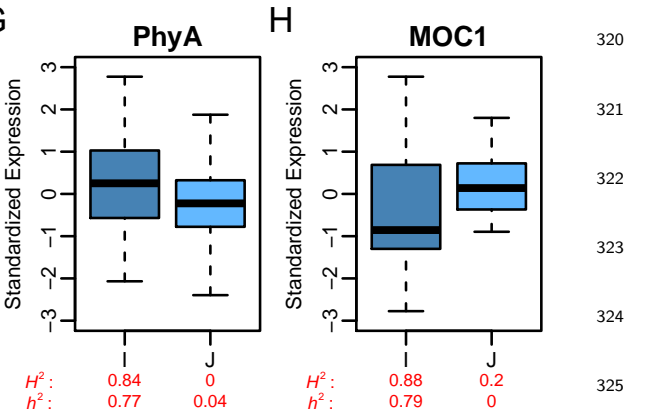


Indica subspecies. A large number of genes were common to both subspecies (21,166 genes). A total of 1,278 334 genes were found to be uniquely expressed in Japonica, and 902 were found to be uniquely expressed in 335 Indica.

A total of 5,005 genes exhibited significant $H^{2}$ in Indica and 3,338 genes in Japonica $(F D R<0.001 ;$ Supplemental File S3). For these genes, $H^{2}$ ranged from 0.67 to 0.98 in Indica and 0.67 to 0.97 in Japonica. 338 A larger number of genes were identified with significant additive genetic variance, with 6,804 identified in 339 Indica and 5,103 found in Japonica. For these genes, narrow-sense heritability ranged from 0.201 to 0.953 in 340 Indica and 0.220 to 0.948 in Japonica. Interestingly, few genes showed significant heritable expression in both ${ }_{341}$ subspecies. For instance, only 1,681 and 2,644 genes were found to have significant $H^{2}$ and $h^{2}$, respectively, ${ }_{342}$ in both Indica and Japonica. Moreover, a comparison of $H^{2}$ and $h^{2}$ between subspecies showed that for ${ }_{343}$ many genes, heritability estimates were considerably different between Indica and Japonica (Figure 44. 344

To systematically identify genes showing significant differences in $H^{2}$ or $h^{2}\left(\Delta H^{2}\right.$ and $\Delta h^{2}$, respectively) ${ }_{345}$ between subspecies, accessions were randomly partitioned into two groups of equal size and the difference in 346 heritability was estimated between groups. The resampling approach was repeated 100 times. A total of ${ }_{347}$ 1,860 genes showed significant differences in $H^{2}(p<0.01)$ between the two subspecies, with a minimum ${ }_{348}$ absolute difference in $H^{2}$ of 0.40 . Fewer genes were identified with a significant difference in $h^{2}$ between ${ }_{349}$ Japonica and Indica (Supplemental File S4). Only 1,325 genes were found with significant differences in $h^{2} \quad 350$ between Indica and Japonica, and the absolute difference in $h^{2}$ ranged from 0.54 to 0.95 (Figure 44. 351

These differences in heritability may be due to insufficient phenotypic variation (e.g. lack of expression 352 diversity), or changes in the genetic or environmental factors that contribute to phenotypic variation. Thus, 353 to further examine the potential causes of the observed differences in hertiability, we quantified the 354 expression diversity $(\mathrm{CV})$, genetic variation and environmental variation within each subspecies for genes 355 exhibiting $\Delta H^{2}$ and $\Delta h^{2}$, as well as those with shared heritable variation. For genes exhibiting 356 subspecies-specific genetic variability, the loss of heritability was largely due to an increase in environmental 357 effects on phenotypic variation in the subspecies lacking heritability rather than loss of phenotypic variation. 358 This is clearly evident in Supplemental Figure S2 The mean CV for $\Delta H^{2}$ genes decreased slightly in 359 subspecies lacking genetic variability. However, for these same genes the proportion of phenotypic variation 360 that was explained by environmental effects increased significantly in subspecies lacking genetic variability. 361 Collectively, these results suggest that the differences in heritability exhibited between the subspecies is 362 driven largely by loss of genetic variability and an increase in environmental effects rather than a loss of ${ }_{363}$ phenotypic variation.

Interestingly, several genes that have been reported to have divergent genetic variants between Indica and 365 
Japonica were found within $\Delta H^{2}$ and $\Delta h^{2}$ genes. For instance, DOPPELGANGER1 (DPL1) showed 366 significantly higher $H^{2}$ and $h^{2}$ in Indica relative to Japonica $\left(H^{2}: 0.92\right.$ and 0.27 , respectfully, $p_{\Delta H^{2}}=0.011 ; \quad 367$ $h^{2}: 0.81$ and $\left.0.17, p_{\Delta h^{2}}=0.004 ; 4 \mathrm{E}\right)$. However for DOPPELGANGER2, the converse was true. ${ }_{368}$ Significantly higher $H^{2}$ and $h^{2}$ was observed in Japonica relative to Indica $\left(H^{2}: 0.87\right.$ and $0.03, \quad 369$ $p_{\Delta H^{2}}<0.001 ; h^{2}: 0.77$ and 0, respectfully, $p_{\Delta h^{2}}=0.005$; Figure 4F). Mizuta et al. (2010) showed that 370 $D P L 1$ and DPL2 are important regulators of Indica-Japonica hybrid incompatibility, and non-functional ${ }_{371}$ alleles arose independently for DPL1 and DPL2 within the Indica and Japonica subspecies respectively. $\quad 372$ Thus the results reported by Mizuta et al. (2010) are consistent with the divergent genetic variability in ${ }_{373}$ expression observed in our study. In addition to DPL1 and DPL2, a gene that is important for the ${ }_{374}$ regulation of shoot growth/ architecture, MOC1, also displayed divergent genetic variability between 375 subspecies. MOC1 showed significant differences in both $H^{2}$ and $h^{2}$ (Figure $4 \mathrm{H}$ ). Collectively, these results 376 show that the two subspecies are divergent at the transcriptional and genetic levels. Moreover, many genes 377 exhibit large differences in genetic variability between the Indica and Japonica, suggesting that these genes ${ }_{378}$ may be regulated by divergent genetic mechanisms.

\section{Joint eQTL analysis assesses cis-regulatory divergence between subspecies}

The differences in the narrow-sense heritability between subspecies observed for some genes suggest a 
being the eQTL was

selected for each gene, and the posterior probability for all three configurations (Indica-specific,

Japonica-specific, and across subspecies)

was compared. Of the 5,097 eQTL genes detected,

$80 \%$ (4,077 genes; 3,826 unique SNPs) were detected across subspecies, 18\% (914 genes; 880 unique

SNPs) were detected for Indica accessions, and 2\% (106 genes; 103 unique SNPs) were detected only in Japonica accessions. These results indicate that while a large portion of cis-eQTLs are shared across the two subspecies of cultivated rice, many genes are regulated by unique cis regulatory mechanisms that are specific to the Indica subspecies.

The presence or absence of cis-regulatory variants within a given subspecies may be the result of the unique domestication histories that have shaped Indica and Japonica, and/or driven by environmental adaptation of the wild progenitors from which they were derived. The absence of variation at the eQTL SNP could be due to sampling during differentiation of the wild progenitors or during domestication (e.g. lost purely by chance), or due to selective pressures imposed by the environment or humans. In the case

\section{Signatures of selection are evident among subspecies specific eQTL}

A

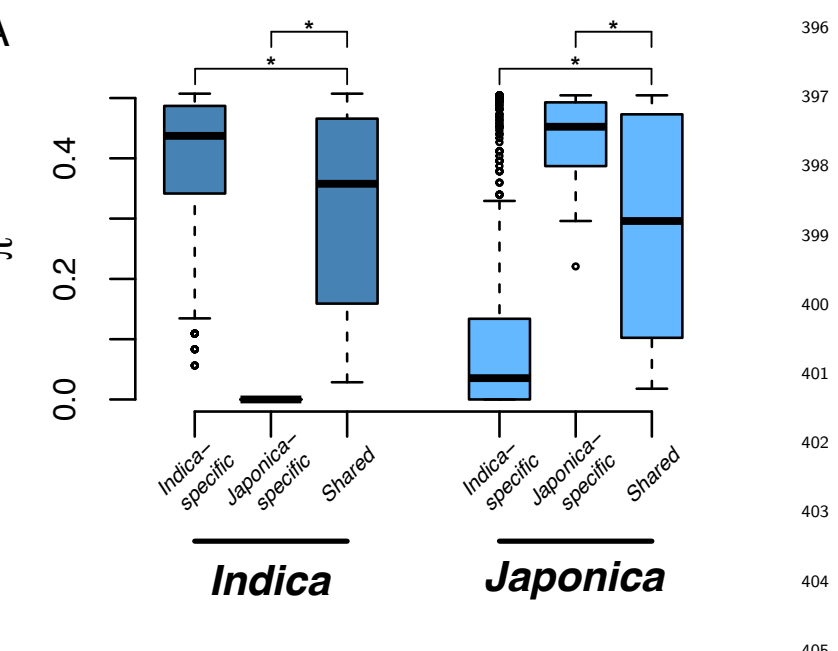

B

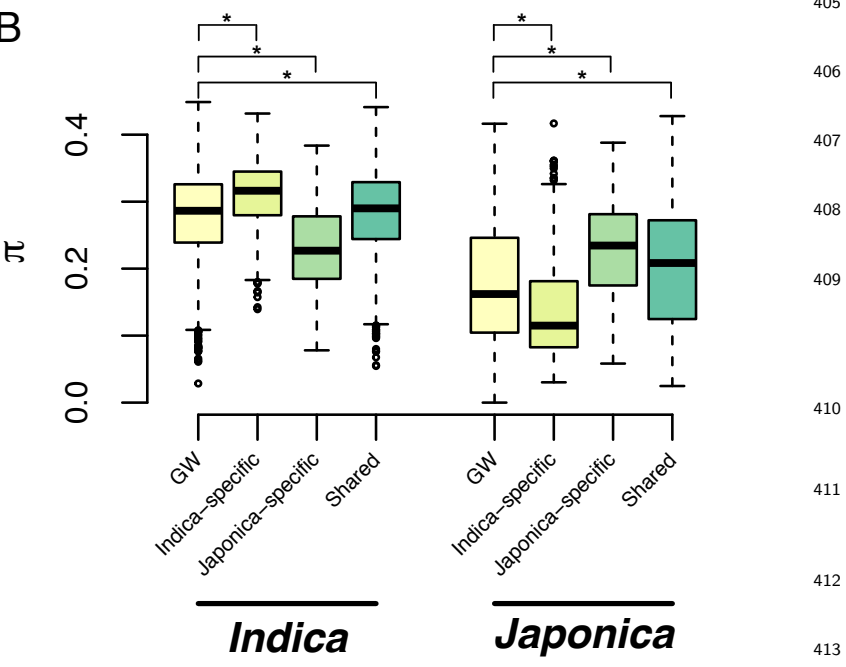

Figure 5. Nucleotide diversity at cis-eQTL. (A) Nucleotide diversity $(\pi)$ for the most significant SNP for each cis-eQTL. The distribution of $\pi$ is pictured fro each subspecies and each eQTL type. (B) Distribution of $\pi$ for $100 \mathrm{~Kb}$ windows around the most significant SNP for each cis-eQTL. Genome-wide (GW) $\pi$ was determined by randomly selecting X SNPs that were more than $100 \mathrm{~kb}$ from a cis-eQTL and low diversity SNPs (MAF < 0.1 in both subspecies) were removed prior to analyses. Asterisks indicate a significant differences determined via Tukey's test between eQTL types $\left(p<1 \times 10^{-8}\right)$.

96

397

398 403 405 406 409 10 413 414 415 416 417 418 419 420 421 of selection, we expect to see reduced genetic diversity around the eQTL compared to the rest of the genome. $\quad{ }^{422}$ To determine whether the absence of subspecies-specific eQTL are the result of selection, we calculated the ${ }^{423}$ average nucleotide diversity $(\pi)$ in $100 \mathrm{~Kb}$ windows around significant subspecies-specific eQTL within each ${ }^{424}$ subspecies and compared these values to the overall average $\pi$ for $100 \mathrm{~Kb}$ windows across the genome within ${ }_{425}$ 
each subspecies using a two-sided $t$-test. Comparisons within each subspecies of $\pi$ for eQTLs and the genome-wide average should account for the inherent differences in $\pi$ between the two subspecies.

Consistent with what would be expected under selection, a significant reduction in nucleotide diversity

Given the small sample size in the current study $(n=91)$ we sought to confirm these results using resequencing data for a larger population of 3,024 diverse rice accessions (Wang et al., 2018; Mansueto et al., $2016 \mathrm{a}$ b, Alexandrov et al. 2014). To this end, we extracted SNP information for 3,024 rice accessions in the same $100 \mathrm{~Kb}$ window surrounding eQTL, and examined $\pi$ within each subpopulation for these regions. As above, $\pi$ within these regions were compared with genome-wide averages for $100 \mathrm{~kb}$ windows. The 3,024 rice accessions are classified into 12 subpopulations: admix (103 accessions), aromatic (76 accessions), aus (201 accessions), indica1A (209 accessions), indica1B (205 accessions), indica2 (285 accessions), indica3 (475 accessions), indica- $X$ (615 accessions), japonica- $X$ (83 accessions), subtropical japonica (112 accessions), temperate japonica (288 accessions), and tropical japonica (372 accessions). The Indica subspecies are represented by indica1 $A$, indica1B, indica2, indica3, and indica- $X$; while the Japonica subspecies consists of the japonica-X, subtropical japonica, temperate japonica, and tropical japonica subpopulations.

Consistent with the results derived from the 91 accessions, $\pi$ within subspecies-specific eQTL was lower in subpopulations lacking the eQTL. For instance, for the Japonica subpopulations (japonica-x, subtropical japonica, temperate japonica, and tropical japonica) $\pi$ estimates for Indica-specific eQTL were considerably lower than those for Indica subpopulations (indica-1A, indica-1B, indica-2, indica-3, and indica-x). The converse was true for Japonica-specific eQTL, with lower $\pi$ observed in Indica subpopulations relative to Japonica. However for the shared eQTL, $\pi$ estimates were higher than the genome-wide averages, suggesting that genetic diversity within regions that regulate gene expression is maintained. 
To identify specific loci that may have been targeted by selection, we selected eQTL regions with an average $\pi$ within a $100 \mathrm{~Kb}$ window that was below the the $5 \%$ quantile for genome-wide average for a given subspecies. Consistent with the results above, we observed a greater frequency of low diversity eQTL the subspecies-specific eQTL. For instance, approximately $11 \%$ of the 880 Indica-specific eQTL were found

in regions of low diversity

in Japonica $\left(\pi_{J a p} \leq 0.0645\right)$.

While for Japonica-specific

eQTL, $14 \%$ (14

of the 103) eQTL regions were lying in regions of low diversity in Indica $\left(\pi_{\text {Ind }} \leq 0.1617\right)$.

However, for shared eQTL and for subspecies in which the subspecies-specific eQTL was detected, the converse was true. Only a small percentage of regions in subspecies lacking
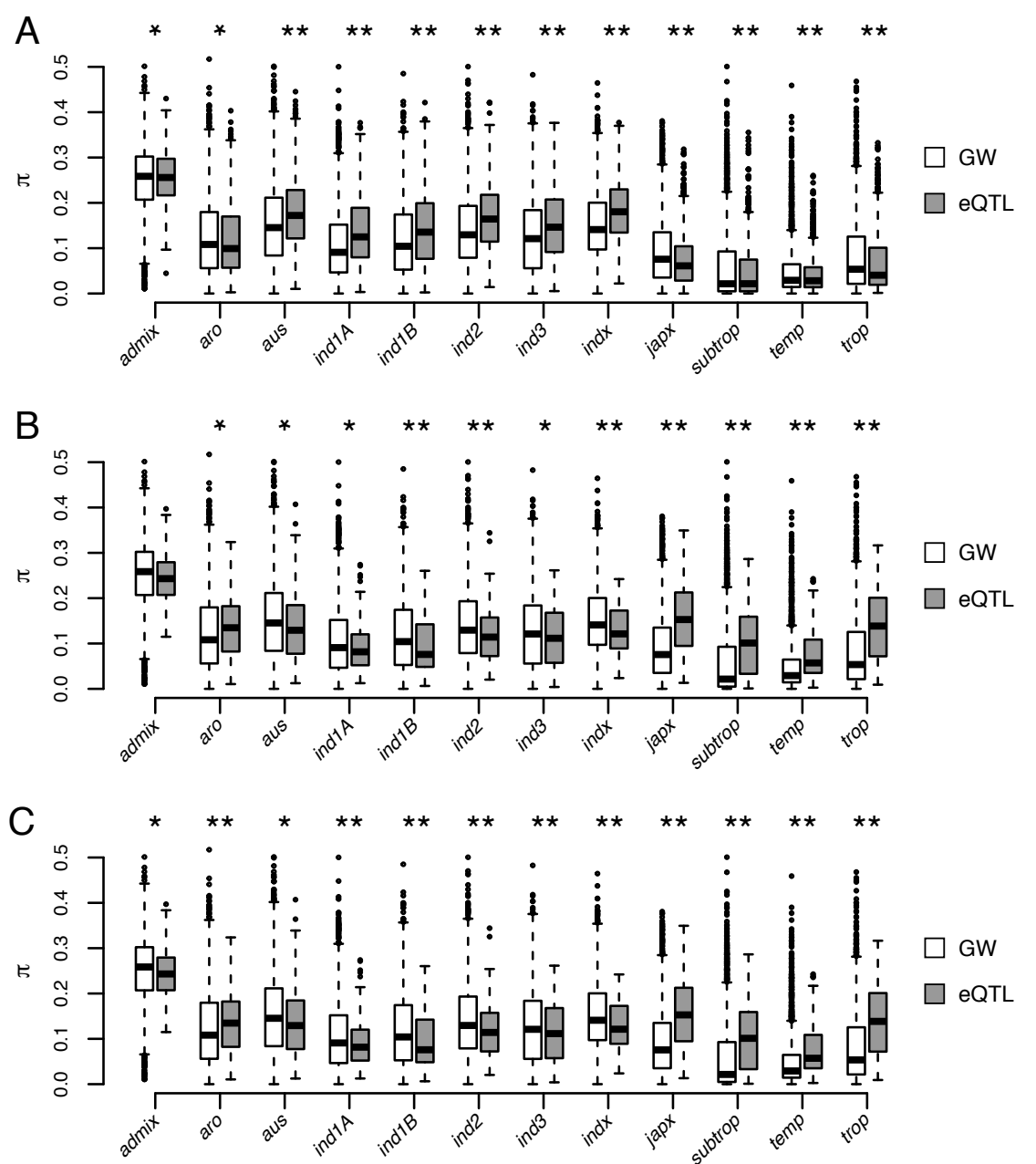

Figure 6. Nucleotide diversity at cis-eQTL within subpopulations for 3,053 rice accessions. Average nucleotide diversity $(\pi)$ for $100 \mathrm{~kb}$ regions surrounding Indica-specific, Japonica-specific, and shared eQTL are pictured in panels A, B, and C, respectively. For each, subpopulation and class of eQTL (e.g.Indica-specific, Japonica-specific, and shared) $\pi$ was calculated for each SNP within $100 \mathrm{~kb}$ of the most significant eQTL SNP. $\pi$ for the eQTL windows were compared to a genome wide (GW) average in which regions with eQTL and site with low diversity (MAF $<0.01$ in 10 of 12 subpopulations) were excluded. Asterisks indicate significant differences between GW and eQTL regions determined using a two-sided Student's $t$-test $(* p<0.05 ; * * p<0.001)$. Subpopulations are named following Wang et al. (2018) (aro: aromatic; ind1A: indica-1A; ind1B: indica-1B; ind2: indica-2; indx: indica-X; japx: japonica-X; subtrop: subtropical japonica; temp: temperate japonica; trop: tropical japonica).
eQTL regions were found within regions of low diversity. For instance, approximately $3.5 \%$ of shared eQTL were found in regions of low diversity in both Indica and Japonica, and less than $1 \%$ of subspecies eQTL were found in regions of low diversity in the subspecies in which they were detected. Collectively these results suggest that selective pressures may have shaped the cis-regulatory divergence of the Indica and

484

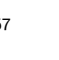

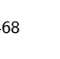

469

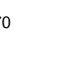

472

"s

.

.

478

.

-

.

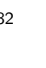




\section{Discussion}

The differentiation between the Indica and Japonica subspecies of cultivated rice has been intensively studied

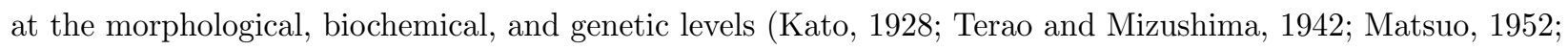

The transcriptional divergence is most evident in the large number of expressed genes showing differences in the magnitude or frequency of expression. Of the 25,732 genes showing evidence of expression in the of Indica and Japonica, recent studies have utilized whole genome sequencing to shed light on the genetic differentiation between the subspecies of cultivated rice (Huang et al., 2012b; Wang et al., 2018). In a recent study, Wang et al. (2018) found that on average approximately $15 \%$ of all genes showed evidence of PAV 


\section{Potential causes of transcriptional divergence between Indica and Japonica}

Lower mean expression values or absence of expression in a given subspecies may be the result of both

heritable and non-heritable effects. The availability of high density SNP information for RDP1 allowed us to ${ }_{518}$ begin to elucidate the genetic basis of the observed transcriptional divergence between the subspecies of 519 cultivated rice. A notable portion of genes with evidence of PAV or DE also showed differences in genetic ${ }_{520}$ variability between the subspecies (13\% and $9 \%$ of DE genes showed differences in $H^{2}$ and $h^{2}$, respectively, ${ }_{521}$ and $20 \%$ and $15 \%$ of PAV genes showed differences in $H^{2}$ and $h^{2}$ ), indicating that for many genes, the ${ }_{522}$ genetic mechanisms that regulate expression may be different between the two subspecies. However, many ${ }_{523}$ genes that display divergent expression patterns have non-significant differences in genetic variability. There ${ }_{524}$ are several explanations for this. For one, the thresholds used to identify genetically divergent genes were ${ }_{525}$ quite stringent. For instance, genes must have a difference in genetic variability in either the broad sense ${ }_{526}$ greater than 0.4022 between subspecies to be labeled as statistically significant, and in the narrow sense ${ }_{527}$ 0.5364. Therefore, it is possible that many more DE or PAV genes have different genetic architectures in the ${ }_{528}$ two subspecies, but were missed because of the statistical threshold selected. A second possibility is that ${ }_{529}$ many of the genes showed divergent expression are influenced greatly by the environment, and thus have low ${ }_{530}$ heritability. Thus, these genes would be filtered out in these genetic analyses.

The heritable transcriptional divergence may be due to genetic variants that influence gene expression and are divergent between Indica and Japonica. These include large structural variants (e.g. deletions, insertions, inversions, and/or duplications), or SNPs that may act in cis or trans to influence gene regulation. While high density SNP information is available for this population and can be leveraged to identify SNPs that regulate expression and are divergent between the subspecies, the identification of larger structural variants that influence expression is only attainable through full genome sequencing, which is not currently available for RDP1. As more genetic resources become available for RDP1 this would be a promising future direction to resolve the causal basis of these transcriptional differences.

The availability of high density SNP information for RDP1 allowed us to begin to elucidate the genetic basis of the observed transcriptional divergence between the subspecies of cultivated rice, and classify genetic effects into those that are common between subspecies, or unique to a given subspecies. While the eQTL-BMA approach has proven to be a powerful framework for assessing the specificity of eQTL for a given tissue or population, one potential limitation of eQTL-BMA is that the framework only allows modeling cis-eQTL. Trans-eQTLs are often difficult to detect due the penalties associated with the large number of statistical tests performed, and because trans-eQTL often have small effect sizes and thus require 
larger dataset for detection. Several studies in humans have shown that cis-eQTL typically only explain ${ }^{54}$ $30-40 \%$ of genetic variation in expression (Price et al., 2011, Grundberg et al., 2012, Hore et al., 2016). Thus, ${ }_{548}$ the divergent regulatory variants captured in the current study only reflect a portion of the differences in ${ }_{549}$ genetic variation between the two subspecies. Further studies are necessary to shed light on the contribution ${ }_{550}$ of trans-regulatory variants on the genetic differentiation between Indica and Japonica transcriptomes. ${ }_{551}$

The joint eQTL analysis facilitated the identification of 5,097 genes associated with one or more SNP in $\quad{ }_{552}$ cis. For most of these genes (81\%), the cis-regulatory variant was shared between both subspecies, indicating ${ }_{553}$ that much of the cis-regulatory variation is common between the two subspecies. This high degree of overlap ${ }_{554}$ is somewhat expected. For one, both Indica and Japonica originate from populations of the same species, ${ }_{555}$ Oryza rufipogon. Moreover, crosses between Indica and Japonica often produce viable offspring, indicating a 556 high degree of colinearity and functional similarity between the genomes. Thus, while considerable ${ }_{557}$ differentiation between founder Oryza rufipogon populations has been reported and further divergence has ${ }_{558}$ likely occurred since domestication, the common origin and inter-specific comparability suggests that the ${ }_{559}$ transcriptional regulation and genome structure is similar (Huang et al., 2012b).

Despite the majority of cis-regulatory variants being shared between the two subspecies, approximately $18 \%$ of all genes with one or more eQTL were found to be unique to Indica or Japonica. The large majority of these subspecies-specific eQTL were detected in the Indica subspecies and were nearly fixed in Japonica indicating low genetic diversity at the eQTL. Moreover, the genetic variation surrounding subspecies-specific eQTL were significantly lower that genome wide averages, indicating that selective pressures may have uniquely shaped components of cis-regulatory variation between the two subspecies. The two subspecies are derived from geographically and genetically distinct subpopulations of Oryza rufipogon (Huang et al., 2012b). Therefore, it remains an open question whether these events occurred during the differentiation between $O$. rufipogon subpopulations or during the domestication of $O$. sativa.

We found significantly higher nucleotide diversity in the regions surrounding eQTL compared to genome wide averages. These patterns of diversity were consistent within subpopulations for shared eQTL, as well as for subspecies-specific eQTL in the subspecies or subpopulations in which they were detected. Although the functions for the majority of these eQTL genes are unknown, the observation that their expression is regulated at a genetic level suggests that they may play a role in the regulation of some biological process. Genetic diversity is a prerequisite to evolutionary change (Lewontin et al., 1974). Therefore the higher nucleotide diversity at these regions compared to genome-wide backgrounds may be reflective of the importance of maintaining genetic variation for these biological processes through regulation at the transcriptional level. 


\section{Functional significance of transcriptional divergence}

The current study sheds light on the transcription divergence between the major subspecies of cultivated rice. ${ }_{580}$ Many of these genes found to have divergent expression, genetic variability, or regulatory variation have been ${ }_{581}$ reported to be underlying important agronomic traits, such as photoperiod adaptation and development. 582 Therefore these observed differences may have potential agronomic significance.

Among these divergent genes, we identified three genes (OsPhyA, OsPhyC, and OsCO3), that have been reported to be associated with the timing of reproductive development in response to day length that had significant heritability in Indica only. The two phytochrome genes, OsPhyA and OsPhyC are activated under long-day conditions and repress flowering time through OsGhd7 (Takano et al., 2005, Lee et al., 2016). Although no studies have shown whether OsCO3 participate directly in the pathway involving OsPhy genes, disruption of $\mathrm{OsCO} 3$ interferes with photoperiod sensitivity and/or flowering time (Kim et al., 2008). For instance, Kim et al. (2008) showed that the overexpression of $\mathrm{OsCO} 3$ delayed flowering under short-day

In addition to genes regulating phenology, several genes were identified that have been reported to play important roles in the regulation of shoot architecture $(D 18, M T 2 b$, and $M O C 1)$. For instance, two genes dwarf18 (D18) and Metallothionein2b (MT2b) have been reported to regulate plant height (Itoh et al. 2001; Yuan et al. 2008). D18 encodes a GA- $\beta$ hydroxylase and is involved with GA biosynthesis. Loss of $p=0.19$, respectively), indicating that genetic differences may be confined to local regions around $D 18$. The ${ }_{604}$ diversity within the $100 \mathrm{~kb}$ regions surrounding the eQTL region was quite high compared to the genome-wide average in both subspecies $\left(\pi_{\text {Ind }}=0.27, \pi_{\text {Jap }}=0.18\right)$ indicating that the absence of the $D 18{ }_{606}$ eQTL in Japonica may be due to low diversity within the eQTL SNP, rather than potential selective 607 pressures between subspecies. 


\section{Conclusions}

The morphological and genetic differences between subspecies of cultivated rice have been studied extensively, ${ }_{610}$ however the divergence of Indica and Japonica at the transcriptional and regulatory levels is largely unresolved. Here, we provide, to date, the first detailed population-level characterization of transcriptional 


\section{Acknowledgments}

\section{Data Availability}

All transcriptional data can be accessed via NCBI Gene Expression Omnibus under accession number 


\section{References}

Alexandrov, N., Tai, S., Wang, W., Mansueto, L., Palis, K., Fuentes, R. R., Ulat, V. J., Chebotarov, D., Zhang, G., Li, Z., et al. (2014). Snp-seek database of snps derived from 3000 rice genomes. Nucleic acids research, 43(D1):D1023-D1027.

Anders, S., Pyl, P. T., and Huber, W. (2015). Htseq - a python framework to work with high-throughput sequencing data. Bioinformatics, 31(2):166-169.

Andrews, S. et al. (2010). FastQC: a quality control tool for high throughput sequence data.

Bates, D., Mächler, M., Bolker, B., and Walker, S. (2015). Fitting linear mixed-effects models using lme4. Journal of Statistical Software, 67(1):1-48.

Benjamini, Y. and Hochberg, Y. (1995). Controlling the false discovery rate: a practical and powerful approach to multiple testing. Journal of the royal statistical society. Series B (Methodological), pages 289-300.

Bolger, A. M., Lohse, M., and Usadel, B. (2014). Trimmomatic: a flexible trimmer for illumina sequence data. Bioinformatics, 30(15):2114-2120.

Butler, D., Cullis, B. R., Gilmour, A., and Gogel, B. (2009). Asreml-r reference manual. The State of Queensland, Department of Primary Industries and Fisheries, Brisbane.

Caicedo, A. L., Williamson, S. H., Hernandez, R. D., Boyko, A., Fledel-Alon, A., York, T. L., Polato, N. R., Olsen, K. M., Nielsen, R., McCouch, S. R., et al. (2007). Genome-wide patterns of nucleotide polymorphism in domesticated rice. PLoS genetics, 3(9):e163.

Danecek, P., Auton, A., Abecasis, G., Albers, C. A., Banks, E., DePristo, M. A., Handsaker, R. E., Lunter, G., Marth, G. T., Sherry, S. T., et al. (2011). The variant call format and vcftools. Bioinformatics, $27(15): 2156-2158$.

Ding, J., Araki, H., Wang, Q., Zhang, P., Yang, S., Chen, J.-Q., and Tian, D. (2007). Highly asymmetric rice genomes. BMC genomics, 8(1):154.

Eizenga, G. C., Ali, M., Bryant, R. J., Yeater, K. M., McClung, A. M., McCouch, S. R., et al. (2014). Registration of the rice diversity panel 1 for genomewide association studies. Journal of Plant Registrations, 8(1):109-116. 
Famoso, A. N., Zhao, K., Clark, R. T., Tung, C.-W., Wright, M. H., Bustamante, C., Kochian, L. V., and McCouch, S. R. (2011). Genetic architecture of aluminum tolerance in rice (Oryza sativa) determined through genome-wide association analysis and QTL mapping. PLoS genetics, 7(8):e1002221.

Feltus, F. A., Wan, J., Schulze, S. R., Estill, J. C., Jiang, N., and Paterson, A. H. (2004). An snp resource for rice genetics and breeding based on subspecies indica and japonica genome alignments. Genome research, 14(9):1812-1819.

Flutre, T., Wen, X., Pritchard, J., and Stephens, M. (2013). A statistical framework for joint eqtl analysis in multiple tissues. PLoS genetics, 9(5):e1003486.

Garris, A. J., Tai, T. H., Coburn, J., Kresovich, S., and McCouch, S. (2005). Genetic structure and diversity in Oryza sativa L. Genetics, 169(3):1631-1638.

Glaszmann, J.-C. (1987). Isozymes and classification of asian rice varieties. Theoretical and Applied Genetics, $74(1): 21-30$.

Goff, S. A., Ricke, D., Lan, T.-H., Presting, G., Wang, R., Dunn, M., Glazebrook, J., Sessions, A., Oeller, P., Varma, H., et al. (2002). A draft sequence of the rice genome (Oryza sativa L. ssp. japonica). Science, 296(5565):92-100.

Grundberg, E., Small, K. S., Hedman, Å. K., Nica, A. C., Buil, A., Keildson, S., Bell, J. T., Yang, T.-P., Meduri, E., Barrett, A., et al. (2012). Mapping cis-and trans-regulatory effects across multiple tissues in twins. Nature genetics, 44(10):1084.

Hore, V., Viñuela, A., Buil, A., Knight, J., McCarthy, M. I., Small, K., and Marchini, J. (2016). Tensor decomposition for multiple-tissue gene expression experiments. Nature genetics, 48(9):1094.

Huang, C.-L., Hung, C.-Y., Chiang, Y.-C., Hwang, C.-C., Hsu, T.-W., Huang, C.-C., Hung, K.-H., Tsai, K.-C., Wang, K.-H., Osada, N., et al. (2012a). Footprints of natural and artificial selection for photoperiod pathway genes in Oryza. The Plant Journal, 70(5):769-782.

Huang, X., Kurata, N., Wang, Z.-X., Wang, A., Zhao, Q., Zhao, Y., Liu, K., Lu, H., Li, W., Guo, Y., et al. (2012b). A map of rice genome variation reveals the origin of cultivated rice. Nature, 490(7421):497.

Huang, X., Lu, G., Zhao, Q., Liu, X., and Han, B. (2008). Genome-wide analysis of transposon insertion polymorphisms reveals intraspecific variation in cultivated rice. Plant physiology, 148(1):25-40. 
Huang, X., Sang, T., Zhao, Q., Feng, Q., Zhao, Y., Li, C., Zhu, C., Lu, T., Zhang, Z., Li, M., et al. (2010). Genome-wide association studies of 14 agronomic traits in rice landraces. Nature genetics, 42(11):961.

Itoh, H., Tatsumi, T., Sakamoto, T., Otomo, K., Toyomasu, T., Kitano, H., Ashikari, M., Ichihara, S., and Matsuoka, M. (2004). A rice semi-dwarf gene, tan-ginbozu (d35), encodes the gibberellin biosynthesis enzyme, ent-kaurene oxidase. Plant molecular biology, 54(4):533-547.

Itoh, H., Ueguchi-Tanaka, M., Sentoku, N., Kitano, H., Matsuoka, M., and Kobayashi, M. (2001). Cloning and functional analysis of two gibberellin $3 \beta$-hydroxylase genes that are differently expressed during the growth of rice. Proceedings of the National Academy of Sciences, 98(15):8909-8914.

Jung, K.-H., Gho, H.-J., Giong, H.-K., Chandran, A. K. N., Nguyen, Q.-N., Choi, H., Zhang, T., Wang, W., Kim, J.-H., Choi, H.-K., et al. (2013). Genome-wide identification and analysis of japonica and indica cultivar-preferred transcripts in rice using 983 affymetrix array data. Rice, 6(1):19.

Kato, A. (1928). On the affinity of rice varieties as shown by the fertility of rice plants. Centr. Agric. Inst. Kyushu Imp. Univ., 2:241-276.

Kim, S.-K., Yun, C.-H., Lee, J. H., Jang, Y. H., Park, H.-Y., and Kim, J.-K. (2008). Osco3, a constans-like gene, controls flowering by negatively regulating the expression of ft-like genes under sd conditions in rice. Planta, 228(2):355.

Koide, Y., Ogino, A., Yoshikawa, T., Kitashima, Y., Saito, N., Kanaoka, Y., Onishi, K., Yoshitake, Y., Tsukiyama, T., Saito, H., et al. (2018). Lineage-specific gene acquisition or loss is involved in interspecific hybrid sterility in rice. Proceedings of the National Academy of Sciences, 115(9):E1955-E1962.

Lee, Y.-S., Yi, J., and An, G. (2016). Osphya modulates rice flowering time mainly through osgi under short days and ghd7 under long days in the absence of phytochrome b. Plant molecular biology, 91(4-5):413-427.

Lewontin, R. C. et al. (1974). The genetic basis of evolutionary change, volume 560. Columbia University Press New York.

Love, M. I., Huber, W., and Anders, S. (2014). Moderated estimation of fold change and dispersion for rna-seq data with deseq2. Genome biology, 15(12):550.

Lu, T., Lu, G., Fan, D., Zhu, C., Li, W., Zhao, Q., Feng, Q., Zhao, Y., Guo, Y., Li, W., et al. (2010). Function annotation of rice transcriptome at single nucleotide resolution by rna-seq. Genome research, pages gr-106120. 
Mansueto, L., Fuentes, R. R., Borja, F. N., Detras, J., Abriol-Santos, J. M., Chebotarov, D., Sanciangco, M., Palis, K., Copetti, D., Poliakov, A., et al. (2016a). Rice snp-seek database update: new snps, indels, and queries. Nucleic acids research, 45(D1):D1075-D1081.

Mansueto, L., Fuentes, R. R., Chebotarov, D., Borja, F. N., Detras, J., Abriol-Santos, J. M., Palis, K., Poliakov, A., Dubchak, I., Solovyev, V., et al. (2016b). SNP-Seek II: A resource for allele mining and analysis of big genomic data in Oryza sativa. Current Plant Biology, 7:16-25.

Mather, K. A., Caicedo, A. L., Polato, N., Olsen, K. M., McCouch, S., and Purugganan, M. D. (2007). The extent of linkage disequilibrium in rice (oryza sativa 1.). Genetics.

Matsuo, T. (1952). Genecological studies on cultivated rice. Bull. Natl. Inst. Agr. Sci. Jpn. D, 3:1-111.

McCouch, S. R., Wright, M. H., Tung, C.-W., Maron, L. G., McNally, K. L., Fitzgerald, M., Singh, N., DeClerck, G., Agosto-Perez, F., Korniliev, P., et al. (2016). Open access resources for genome-wide association mapping in rice. Nature communications, 7:10532.

Mizuta, Y., Harushima, Y., and Kurata, N. (2010). Rice pollen hybrid incompatibility caused by reciprocal gene loss of duplicated genes. Proceedings of the National Academy of Sciences, 107(47):20417-20422.

Morinaga, T. (1954). Classification of rice varieties on the basis of affinity. Jpn. J. Breed., 4:1-14.

Morishima, H. and Oka, H.-I. (1981). Phylogenetic differentiation of cultivated rice, xxii. numerical evaluation of the indica-japonica differentiation. Japanese Journal of Breeding, 31(4):402-413.

Naranjo, L., Talón, M., and Domingo, C. (2014). Diversity of floral regulatory genes of japonica rice cultivated at northern latitudes. BMC genomics, 15(1):101.

Oka, H. et al. (1991). Genetic diversity of wild and cultivated rice. Rice biotechnology, pages 55-81.

Price, A. L., Helgason, A., Thorleifsson, G., McCarroll, S. A., Kong, A., and Stefansson, K. (2011).

Single-tissue and cross-tissue heritability of gene expression via identity-by-descent in related or unrelated individuals. PLoS genetics, 7(2):e1001317.

Pritchard, J. K., Stephens, M., and Donnelly, P. (2000). Inference of population structure using multilocus genotype data. Genetics, 155(2):945-959.

Schatz, M. C., Maron, L. G., Stein, J. C., Wences, A. H., Gurtowski, J., Biggers, E., Lee, H., Kramer, M., Antoniou, E., Ghiban, E., et al. (2014). Whole genome de novo assemblies of three divergent strains of rice, Oryza sativa, document novel gene space of aus and indica. Genome biology, 15(11):506. 
Scheipl, F., Greven, S., and Kuechenhoff, H. (2008). Size and power of tests for a zero random effect variance or polynomial regression in additive and linear mixed models. Computational Statistics Data Analysis, $52(7): 3283-3299$.

Song, Y. H., Shim, J. S., Kinmonth-Schultz, H. A., and Imaizumi, T. (2015). Photoperiodic flowering: time measurement mechanisms in leaves. Annual review of plant biology, 66:441-464.

Stein, J. C., Yu, Y., Copetti, D., Zwickl, D. J., Zhang, L., Zhang, C., Chougule, K., Gao, D., Iwata, A., Goicoechea, J. L., et al. (2018). Genomes of 13 domesticated and wild rice relatives highlight genetic conservation, turnover and innovation across the genus Oryza. Nature genetics, 50(2):285.

Takano, M., Inagaki, N., Xie, X., Yuzurihara, N., Hihara, F., Ishizuka, T., Yano, M., Nishimura, M., Miyao, A., Hirochika, H., et al. (2005). Distinct and cooperative functions of phytochromes a, b, and c in the control of deetiolation and flowering in rice. The Plant Cell, 17(12):3311-3325.

Terao, H. and Mizushima, U. (1942). Some considerations on the classification of Oryza sativa L. into two subspecies, so called Japonica and Indica. Jpn. J. Bot., 10:213-258.

Trapnell, C., Pachter, L., and Salzberg, S. L. (2009). Tophat: discovering splice junctions with rna-seq. Bioinformatics, 25(9):1105-1111.

VanRaden, P. M. (2008). Efficient methods to compute genomic predictions. Journal of dairy science, 91(11):4414-4423.

Walia, H., Wilson, C., Zeng, L., Ismail, A. M., Condamine, P., and Close, T. J. (2007). Genome-wide transcriptional analysis of salinity stressed japonica and indica rice genotypes during panicle initiation stage. Plant molecular biology, 63(5):609-623.

Wang, W., Mauleon, R., Hu, Z., Chebotarov, D., Tai, S., Wu, Z., Li, M., Zheng, T., Fuentes, R. R., Zhang, F., et al. (2018). Genomic variation in 3,010 diverse accessions of asian cultivated rice. Nature, 557(7703):43.

Wang, X., Kudrna, D. A., Pan, Y., Wang, H., Liu, L., Lin, H., Zhang, J., Song, X., Goicoechea, J. L., Wing, R. A., et al. (2014). Global genomic diversity of Oryza sativa varieties revealed by comparative physical mapping. Genetics, pages genetics-113.

Yoshida, S., Forno, D., Cock, J., and Gomez, K. (1976). Laboratory manual for physiological studies of rice, 3rd edn manila: International rice research institute. 
Yu, J., Hu, S., Wang, J., Wong, G. K.-S., Li, S., Liu, B., Deng, Y., Dai, L., Zhou, Y., Zhang, X., et al. (2002).

A draft sequence of the rice genome (Oryza sativa L. ssp. indica). Science, 296(5565):79-92.

Yuan, J., Chen, D., Ren, Y., Zhang, X., and Zhao, J. (2008). Characteristic and expression analysis of a metallothionein gene, osmt2b, down-regulated by cytokinin suggests functions in root development and seed embryo germination of rice. Plant Physiology, 146(4):1637-1650.

Zhao, K., Tung, C.-W., Eizenga, G. C., Wright, M. H., Ali, M. L., Price, A. H., Norton, G. J., Islam, M. R., Reynolds, A., Mezey, J., et al. (2011). Genome-wide association mapping reveals a rich genetic architecture of complex traits in Oryza sativa. Nature communications, 2:467. 


\section{Supplemental Data}

Table S1. Gene onotology (GO) enrichment analysis for genes exhibiting significant presenceabsence expression variation $(\mathrm{PAV})(F D R<0.001)$. GO enrichment was conducted using AgriGO (http://bioinfo.cau.edu.cn/agriGO) using the MSU V7 genome build without transposable elements as a background. GO enrichment was conducted separately for genes expressed predominately in each subspecies.

\begin{tabular}{llllllll}
\hline Subspecies & GO term & $\begin{array}{l}\text { Ont. } \\
\text { Cat. }\end{array}$ & GO Description & $\begin{array}{l}\text { No. in } \\
\text { input }\end{array}$ & $\begin{array}{l}\text { No. in } \\
\text { back- } \\
\text { ground }\end{array}$ & -value & $F D R$ \\
\hline \hline Japonica & GO:0006950 P & response to stress & 137 & 4660 & $1.5 \times 1^{-10}$ & $5.2 \times 1^{-8}$ \\
& GO:0050896 P & $\begin{array}{l}\text { response to stimulus } \\
\text { response to biotic }\end{array}$ & 172 & 6928 & $1.0 \times 1^{-7}$ & $1.7 \times 1^{-5}$ \\
& GO:0009607 P & & 1404 & $2.4 \times 1^{-4}$ & $2.7 \times 1^{-2}$ \\
& & & stimulus & & & & \\
& GO:0019825 & $\mathrm{F}$ & oxygen binding & 25 & 390 & $5.0 \times 1^{-8}$ & $4.5 \times 1^{-6}$ \\
& GO:0000166 F & nucleotide binding & 92 & 3490 & $2.4 \times 1^{-5}$ & $1.1 \times 1^{-3}$ \\
& GO:0016740 F & transferase activity & 120 & 5200 & $3.6 \times 1^{-4}$ & $9.6 \times 1^{-3}$ \\
& GO:0003824 F & catalytic activity & 271 & 13508 & $4.2 \times 1^{-4}$ & $9.6 \times 1^{-3}$ \\
& GO:0016301 F & kinase activity & 69 & 2699 & $6.4 \times 1^{-4}$ & $9.6 \times 1^{-3}$ \\
Indica & GO:0019825 F & oxygen binding & 13 & 390 & $1.5 \times 1^{-4}$ & $8.8 \times 1^{-3}$ \\
\hline \hline
\end{tabular}


A

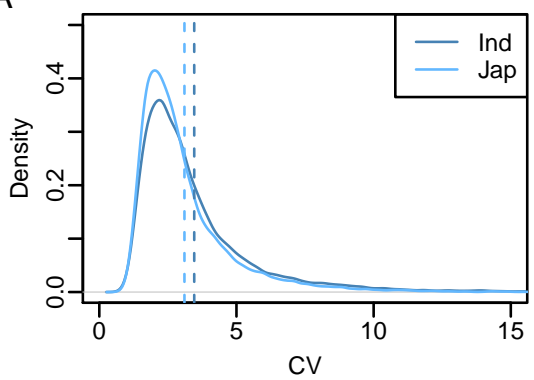

B

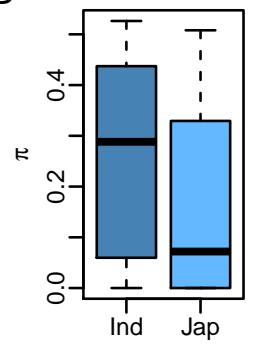

Figure S1. Genetic and expression diversity within Indica and Japonica accessions. (A) The coefficient of variation was used as an estimate of the diversity in gene expression within each subspecies. The vertical dashed lines represent the mean $\mathrm{CV}$ within each subspecies. (B) Site-wise nucleotide diversity $(\pi)$ was used as an estimate of the genetic diversity within each of the subspecies using 36,901 SNPs described by Zhao et al. (2011). 

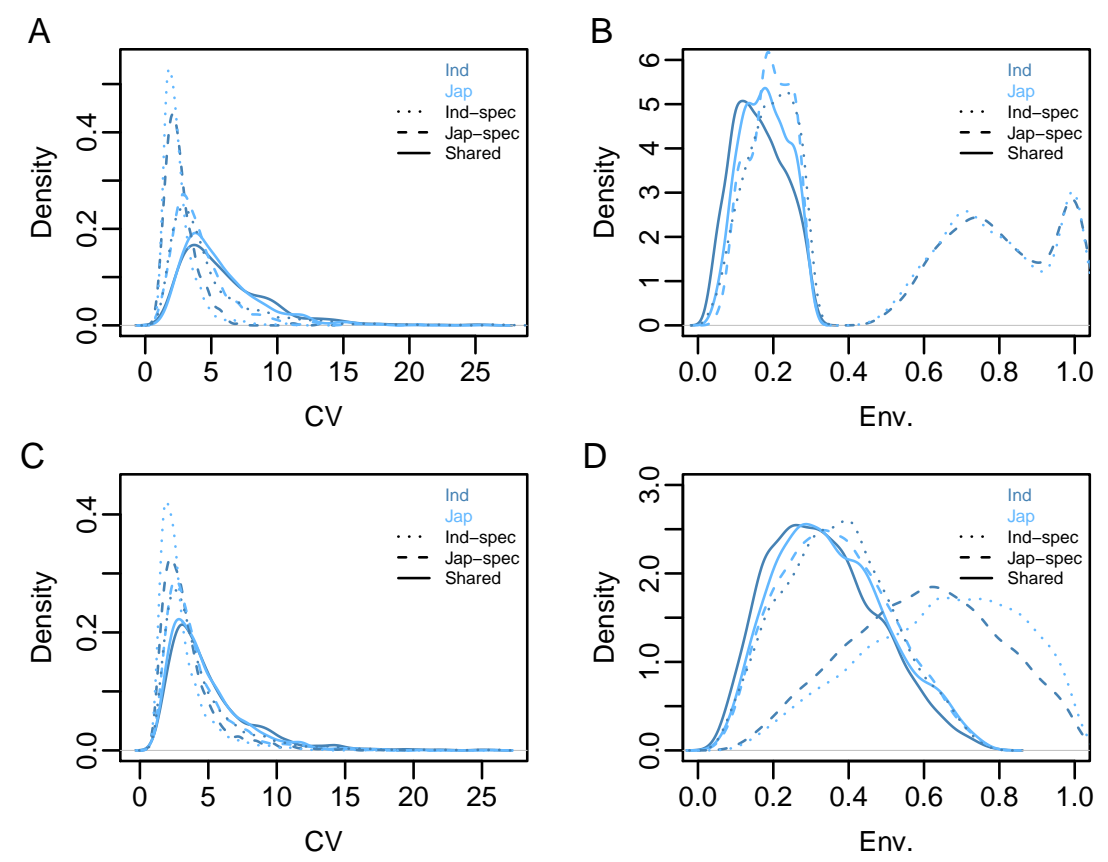

Figure S2. Assessing phenotypic variation and environmental effects for genes exhibiting genetic variability within each subspecies. Genes were classified into three categories based on their patterns of genetic variability. "Shared" refers to genes showing significant genetic variability $(F D R<0.001)$ in both subspecies. The categories "Indicaspecific" and "Japonica-specific" refer to genes that showed significant differences in genetic variability (e.g. $\Delta H^{2}$ or $\Delta h^{2}$ ) and had heritable expression in Indica and Japonica, respectively. Phenotypic variation was assessed using the coefficient of variation (CV) for $H^{2}$ or $h^{2}$ genes (A and C, respectively). The contribution of the environment on phenotypic variation was determined as $1-H^{2}$ and $1-h^{2}$ (B and D, respectively). The categories of genetic variability are indicated by line type, while the subspecies in which CV or environmental variation was measured are indicated by the color of lines. 